\title{
Design Studies for the Radio Neutrino Observatory (RNO)
}

\section{Ben Hokanson-Fasig* for the RNO Collaboration ${ }^{\dagger}$}

University of Wisconsin-Madison

E-mail: fasig@icecube.wisc.edu

The Radio Neutrino Observatory (RNO) is a proposed ultra-high energy neutrino detector that plans to instrument $\sim 100$ square kilometers of ice at the South Pole to study the high-energy end of the cosmic neutrino spectrum. An array of radio-frequency antennas are to be deployed that are sensitive to the Askaryan radio pulses emitted by energetic neutrino-induced cascades in the ice. During the conception of RNO a number of design studies were performed to guide decisions in the collaboration. Ultimately, the balancing of these studies with expected station costs and considerations of installation have determined the layout of the RNO stations. Included in the design studies were tests of effective volume per station, sky coverage, and several event reconstruction quality parameters. The results of these studies point to the current RNO station layout: a group of antennas at a depth of at least 60 meters colocated with a group of near-surface antennas. We will present the results of our design studies and how they justify the proposed station layout of RNO.

36th International Cosmic Ray Conference -ICRC2019-

July 24th - August 1st, 2019

Madison, WI, U.S.A.

\footnotetext{
* Speaker.

${ }^{\dagger}$ for collaboration list see PoS(ICRC2019)1177
} 

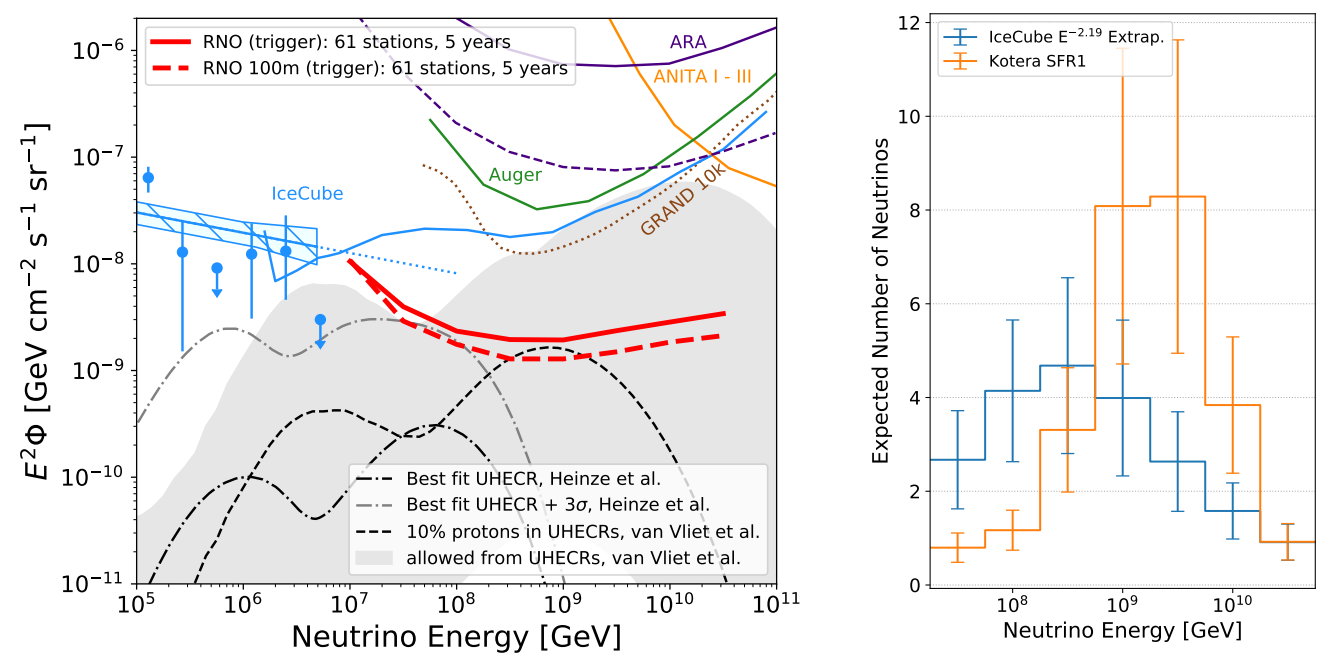

Figure 1: Left: The sensitivity of RNO compared with current experimental results and two diffuse cosmogenic neutrino flux models [8,9]. All limits use one-decade energy binning. Right: The expected number of neutrinos vs. energy for the IceCube $\mathrm{E}^{-2.19}$ extrapolated flux [10] and the Kotera et al. SFR1 flux [11].

\section{Introduction}

The Radio Neutrino Observatory (RNO) has been proposed as a next-generation, ultra-high energy (UHE) neutrino detector to continue the rapid development of radio detection of highenergy cosmic particles over the past decade. The RNO collaboration combines the past expertise of members from the ARA [1, 2], ARIANNA [3, 4], ANITA [5], and RICE [6] experiments. The full detector will offer world-leading measurements of the high-energy neutrino flux and event reconstruction for use in multi-messenger astronomy. In addition, the initial phase of construction for RNO is intended as a pathfinder for the radio component of IceCube-Gen2. [7]

RNO is designed to be an instrument that measures of order ten neutrinos at the highest energies, possibly including the first discovery. At its lowest energies the detector sensitivity will overlap with IceCube measurements, expecting 10-25 astrophysical neutrino events in five years if the measured spectral index extends unbroken to higher energies. Figure 1 compares the sensitivity of RNO with other experiments as well as several models of astrophysical and cosmogenic neutrino production. In the event of a non-detection, RNO will establish the world's best limits at the EeV energy scale. [7]

The detection mechanism used for radio neutrino experiments like RNO is based on coherent, impulsive radio signals that are emitted when any flavor of neutrino interacts in a dense material, due to a negative charge excess in the neutrino-induced cascade. If the wavelength of the radiation is longer than the transverse size of the shower, the resulting emission is coherent, leading to a large boost in total power for high-energy showers at frequencies $\lesssim 1 \mathrm{GHz}$. This is known as the Askaryan effect [12]. Building an observatory for UHE neutrinos around this effect requires instrumenting enormous volumes of a radio-clear dielectric such as glacial ice. The radio attenuation properties of ice have been directly measured at locations in Greenland and Antarctica, showing attenuation lengths in excess of $1 \mathrm{~km}$ at the South Pole [1, 13, 14]. 

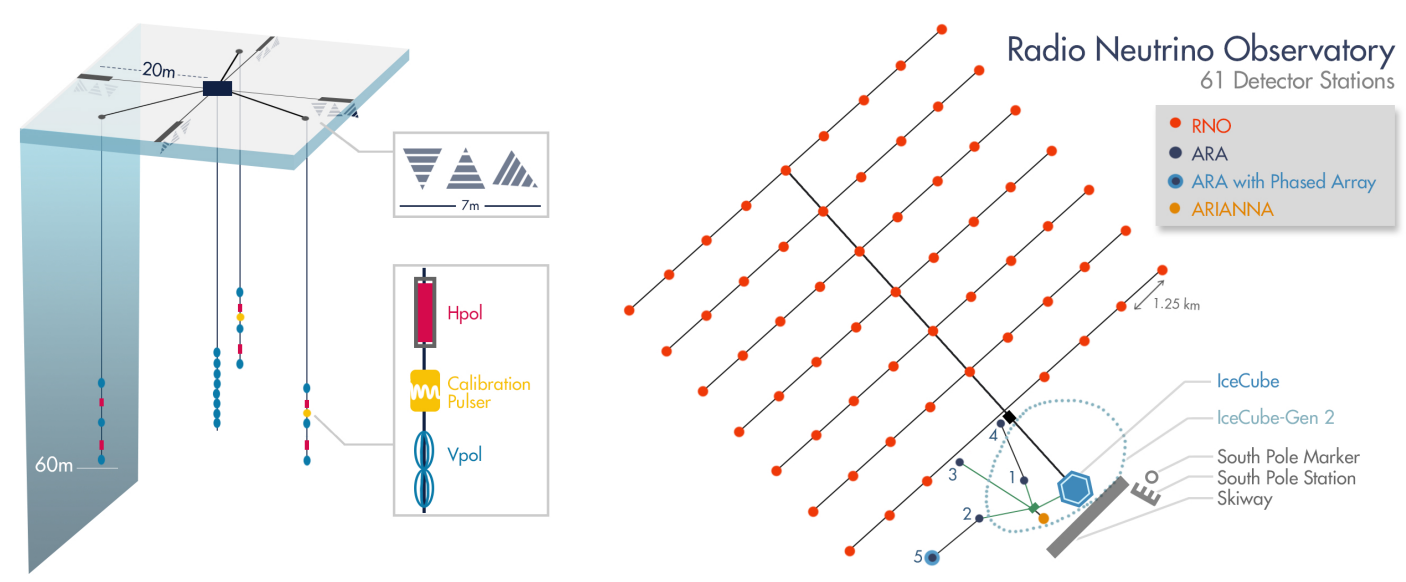

Figure 2: Left: The general layout of each RNO station, consisting of a surface array and a deep array. Right: The layout of the full 61-station array, as viewed from above.

RNO will consist of 61 stations on a grid with an inter-station spacing of $1.25 \mathrm{~km}$. The layout of the individual stations combines an array of sub-surface antennas with another array of deep antennas deployed 60 meters below the surface of the ice, as shown in Figure 2. While the stations were designed with the intention of deployment at the South Pole, deployment in Greenland is also under consideration.

Based on the current landscape of high-energy astrophysics, the following goals for RNO were established as requirements for the completed detector [7]:

1. Achieve sensitivity to neutrinos across a broad range of energies to target both astrophysical and cosmogenic neutrino fluxes, which is best achieved with low trigger thresholds.

2. Achieve high livetimes critical for multi-messenger observations and improved sensitivity to diffuse neutrino fluxes.

3. Provide high-quality energy and direction reconstruction of each neutrino event for multimessenger studies, which is best achieved by comprehensive observations with both a surface and a deep component of a single station.

4. View a large fraction of the sky to enhance multi-messenger observations.

5. Enhance discovery potential by achieving a high reconstruction efficiency of triggered neutrino events and minimizing backgrounds.

The components that can be used to achieve these goals come from the collective expertise of the members of the RNO collaboration. Among the available antenna technologies are log-periodic dipole antennas (LPDAs) which are large high-gain antennas sensitive to broad-band signals polarized in the plane of the antenna [15]. In addition, the available bicone and quad-slot antennas are narrow broad-band antennas which are nearly azimuthally symmetric and sensitive to vertically and horizontally-polarized signals, respectively [1]. Due to their size, the LPDA antennas are preferably deployed near the surface, up to a few meters deep, while the bicone and quad-slot antennas are preferably deployed deep in the ice in holes with a diameter of around $15 \mathrm{~cm}$.

There are also a variety of novel technologies available for the RNO stations, including a data acquisition system capable of handling up to 40 channels and a phased array trigger mechanism. The phased array trigger offers a way of reducing noise in the trigger system in order to trigger at 
lower thresholds. It works by coherently summing the radio waveforms of $N$ antennas with time delays corresponding to a range of angles of incident plane waves. This results in a boost in signalto-noise ratio (SNR) of $\sqrt{N}$, which directly translates into a lower energy threshold for finding neutrinos. By projecting the performance of the existing ARA phased array system to an 8-channel system, the trigger level is expected to reach an SNR value of $1.5 \sigma$ in voltage. [16, 17]

Combining these available components, a baseline design was formed. The baseline station design consists of an array of 8-12 LPDAs just below the surface along with a phased array of 8 bicone antennas deeper in the ice with a wide array of 12-16 bicone and quad-slot antennas around it. The triggering of such a station design would then be driven by the phased array and the surface LPDAs, with antennas in the outer deep array used primarily for reconstruction.

\section{Design Simulations}

Based on previous experience and simulations, the following parameters of the station design were decided. The radius of the stations is 20 meters, compact enough for a single event to trigger many antennas but wide enough to provide a good lever-arm for reconstruction. The surface array consists of LPDAs pointing upwards, downwards, and outwards, allowing for detection of signals in all polarizations. The upwards LPDAs provide the ability to reject cosmic ray events, and allow for calibration with such events [18]. The deep array consists of the phased array and three outer strings of bicone and quad-slot antennas, plus pulsers for calibrating antennas on the other strings. This baseline station design matches the general station layout shown in Figure 2.

Ultimately five designs were considered for a final round of simulations ${ }^{1}$ to determine the depth of the station, which has a large effect on the science potential. The candidate station designs were as follows:

1. A surface-only station consisting of only the surface array with no deep components.

2. A shallow station consisting of the surface array and a phased array at an average depth of 15 meters, with no wider deep array.

3. A station consisting of the surface array, a phased array at an average depth of 50 meters, and a deep array down to 60 meters.

4. A station consisting of the surface array, a phased array at an average depth of 85 meters, and a deep array down to 100 meters.

5. A station consisting of the surface array, a phased array at an average depth of 185 meters, and a deep array down to 200 meters.

\subsection{Effective Volumes}

Each of the candidate stations was simulated for a range of neutrino energies from $10 \mathrm{PeV}$ up to $100 \mathrm{EeV}$. The effective volumes of the stations in this range behave as shown in Figure 3. When the phased array is included in the station it offers a great improvement in the effective volume, an effect that increases with the depth of the phased array, particularly at higher energies.

\footnotetext{
${ }^{1}$ These simulations, including all numbers reported in this proceeding, were done using the next-generation opensource radio simulation package PyREx, version 1.9.0. The simulations were also checked against AraSim, the existing simulation code used by ARA.
} 

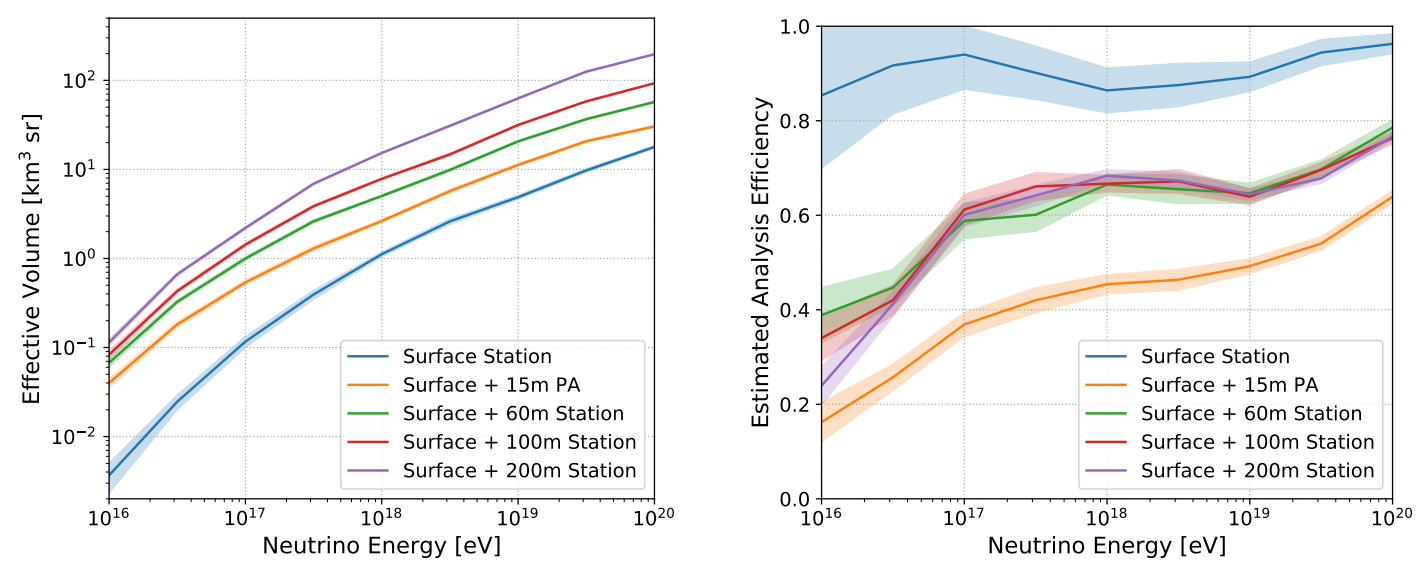

Figure 3: Left: Trigger-level, single-station effective volumes of each station type as a function of energy. Right: Estimate of the analysis efficiency, with statistical error bands. These are calculated as the fraction of triggering events that meet the additional condition of having at least 3 antennas with SNRs greater than $3 \sigma$.

Since the simulated effective volumes are calculated at the trigger level, an attempt was made to estimate the analysis efficiencies of these stations as well, as shown in Figure 3. Based on past experience in reconstructing radio signals, it was determined that requiring 3 antennas to have voltage SNRs above $3 \sigma$ would be a reasonable, if not conservative, proxy for selecting events that would satisfy analysis criteria. The compactness of the surface-only station results in most triggering events satisfying this requirement, but such a station misses out on the increased effective volumes of the stations with a phased array. The difference in analysis efficiency between the 15 meter phased array station and the other deeper stations illustrates the necessity of the outer array of deep antennas for use in reconstruction.

Initial simulations have also been performed for these stations in the Greenland ice as well. Due to the different ice properties, including a shorter attenuation length [14] and different the index of refraction profile [19], the energy dependence of the effective volumes is slightly changed. More studies are warranted to better understand the sensitivity and practical feasibility of an RNO-like detector in Greenland.

\subsection{Sky Coverage}

The position of RNO at the South Pole results in constantly observing the same region of the sky. For this reason, it is important that the stations are designed to observe a fraction of the sky that is as large as possible. If positioned in Greenland instead, the total visible region of the sky would be larger, as the instantaneous field of view is continually in motion due to the rotation of the Earth, but the same source would not be visible at all times. In either case, viewing a large portion of the sky is important for participation in multi-messenger astronomy. Here the visible sky fraction is calculated by the declination band containing $95 \%$ of the triggered events for each station candidate. As can be seen in Figure 5, this visible portion of the sky increases as the station moves deeper in the ice. RNO is planning to develop the necessary tools to play an important role in multi-messenger astronomy, which is expected to be highly important in the coming years, particularly with IceCube's recent event giving a first insight into neutrino sources [20]. 


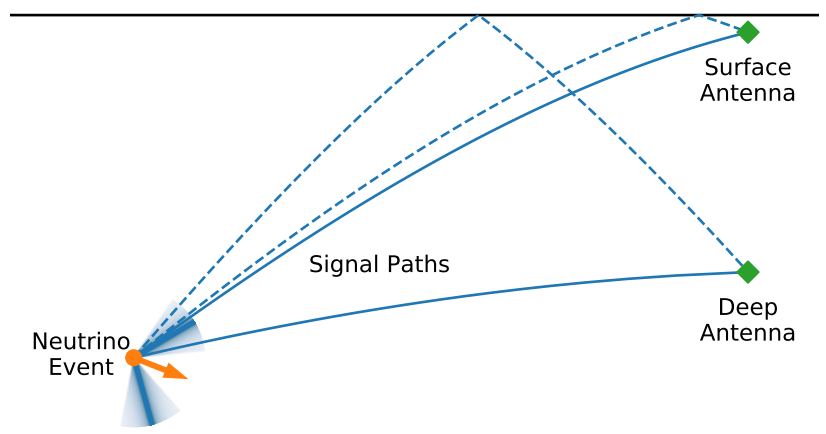

Figure 4: Visualization of signal paths from a neutrino event to surface and deep antennas in the ice. Includes direct paths as solid lines and reflected paths as dashed lines.

\subsection{High-Quality Events}

Beyond the simple analysis proxy outlined above, some events have characteristics that make them particularly useful in analysis. The first class of such events are those with signals detected by both the surface and deep components of the station, as illustrated in Figure 4. This class of events has improved pointing and energy resolution as a result of detecting the signals across a large baseline. The surface coincidence fraction is calculated here as the fraction of triggering events which also have a voltage SNR of at least $3 \sigma$ in any surface antenna. As can be seen in Figure 5, the fraction of events with strong enough signals in both the surface and the deep components decreases as the deep component of the station is moved deeper in the ice.

The second class of highly-analyzable events are so-called "double pulse" events. These events are the result of the fact that the radio signal from a neutrino interaction travels along two paths to each antenna, as shown in Figure 4. Double pulse events are those events where an antenna sees both signals from the direct and reflected (or refracted) paths with a resolvable amplitude. The difference in arrival times of these two paths is a smoking-gun signature for a neutrino event, and is expected to improve the resolution of the neutrino arrival direction. Here the direct + reflected event fraction is calculated as the fraction of triggering events which have direct and reflected pulses with voltage SNRs of at least $1.5 \sigma$ in the phased array or at least $3 \sigma$ in the surface antennas, where the pulses are also separated in time by at least 5 ns. Again, as seen in Figure 5, the fraction of such events decreases as the deep component of the station is moved deeper.

\subsection{Resulting Design}

The design choice for RNO to deploy a station down to 60 meters was informed by balancing the previously mentioned factors in addition to station drilling costs and deployment logistics. Such a station increases both the effective volume per station and the sky coverage, while maintaining a reasonable fraction of "golden events" that can be reconstructed with a higher accuracy. The 60 meter station design also allows for faster and easier deployment than the deeper options, such that all 61 stations could be deployed in four years.

While these station design studies resulted in the decision to deploy 60 meter stations initially, they are open to some interpretation in the relative importance of each factor. The RNO collaboration intends to reconsider this decision as more information comes to light during the initial stages 

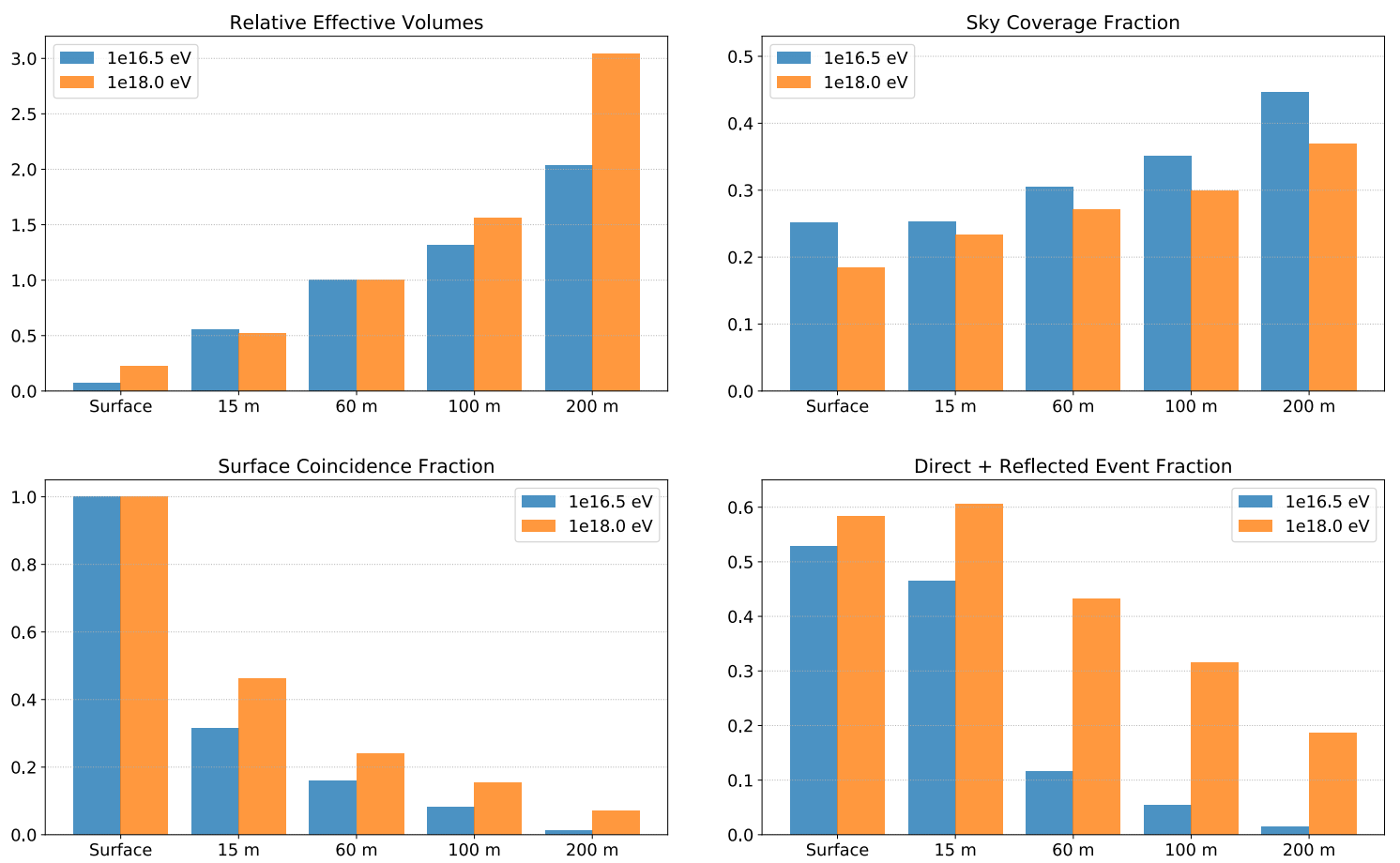

Figure 5: A summary of the factors balanced in the station design study. For simplicity, values are taken for two key energies: $10^{16.5}$ and $10^{18} \mathrm{eV}$. Balancing these factors resulted in the decision to deploy RNO stations at a depth of 60 meters.

of deployment. If for instance there is a stronger science case for maximizing effective area, then later stations may be deployed deeper in the ice. However if there is a requirement for a more complete analysis of a smaller set of events, then later stations may be deployed in a shallower and more compact design. Ultimately the final station design will be decided based on lessons learned on the ice and from data analysis throughout the deployment period.

\section{Summary}

The station design for the Radio Neutrino Observatory (RNO) was formed by referring to past experience as well as simulations of a variety of station layouts. The major design study performed aimed to determine the depth at which the deep portion of the station would be deployed. This study balanced the increased effective volume and sky coverage of deeper stations with the increased event reconstruction quality of shallower stations. The balance between these factors, the station costs, and the ease of deployment was found to be the 60 meter station, with a phased array at 50 meters and an array of LPDAs just below the surface. The relative merits of deploying similar stations in Greenland rather than at South Pole are still under investigation.

With such a design, the 61-station RNO detector will achieve its goals of probing the astrophysical neutrino flux at energies beyond the reach of IceCube and reaching world-leading sensitivity to ultra-high energy neutrinos, with possibilities for multi-messenger astronomy. In the process, RNO will inform the next generation of radio neutrino detection in IceCube-Gen2, providing station designs that are built to deliver the most science for the cost. 


\section{References}

[1] P. Allison et al., Design and Initial Performance of the Askaryan Radio Array Prototype EeV Neutrino Detector at the South Pole, Astropart. Phys. 35, 457 [arXiv:1105.2854].

[2] P. Allison et al., Performance of two Askaryan Radio Array stations and first results in the search for ultra-high energy neutrinos, Phys. Rev. D 93, 082003 [arXiv: 1507.08991 ].

[3] S. W. Barwick et al., A First Search for Cosmogenic Neutrinos with the ARIANNA Hexagonal Radio Array, Astropart. Phys. 70, 12 [arXiv:1410.7352].

[4] S. W. Barwick et al., Performance of the ARIANNA Hexagonal Radio Array, in proceedings of ICRC, POS ( ICRC2015) 1149 (2015).

[5] P. W. Gorham et al., Constraints on the diffuse high-energy neutrino flux from the third flight of ANITA, Phys. Rev. D 98, 022001 [arXiv: 1803.02719$].$

[6] I. Kravchenko et al., RICE Limits on the Diffuse Ultra-High Energy Neutrino Flux, Phys. Rev. D 73, 082002 [arXiv:astro-ph/0601148].

[7] J. A. Aguilar et al., The Next-Generation Radio Neutrino Observatory - Multi-Messenger Neutrino Astrophysics at Extreme Energies, Astro2020 Decadal Survey.

[8] J. Heinze et al., Cosmogenic neutrinos from a combined fit of the Auger spectrum and composition, presented at TeVPA, Berlin (2018).

[9] A. van Vliet et al., Cosmogenic gamma-rays and neutrinos constrain UHECR source models, in proceedings of ICRC, POS ( ICRC2017) 562 (2017).

[10] M. G. Aartsen et al., A Measurement of the Diffuse Astrophysical Muon Neutrino Flux Using Eight Years of IceCube Data, in proceedings of ICRC, POS ( ICRC2 017 ) 1005 (2017).

[11] K. Kotera \& A. V. Olinto, The Astrophysics of Ultrahigh-Energy Cosmic Rays, Annual Review of Astronomy and Astrophysics 49, 119 [arXiv: 1101 . 4256].

[12] G. A. Askar'yan, Excess negative charge of an electron-photon shower and its coherent radio emission, Sov. Phys. JETP 14, 441.

[13] S. W. Barwick et al., South Polar in situ radio-frequency ice attenuation, Journal of Glaciology 51, 231.

[14] J. Avva et al., An in situ measurement of the radio-frequency attenuation in ice at Summit Station, Greenland, Journal of Glaciology 61, 1005.

[15] S. W. Barwick et al., Time Domain Response of the ARIANNA Detector, Astropart. Phys. 62, 139 [arXiv:1406.0820].

[16] A. G. Vieregg et al., A Technique for Detection of PeV Neutrinos Using a Phased Radio Array, JCAP 2,005 [arXiv:1504.08006].

[17] J. Avva et al., Development Toward a Ground-Based Interferometric Phased Array for Radio Detection of High Energy Neutrinos, Nucl. Instrum. Meth. A 869, 46 [arXiv: 1605.03525$].$

[18] S. W. Barwick et al., Radio detection of air showers with the ARIANNA experiment on the Ross Ice Shelf, Astropart. Phys. 90, 50 [arXiv: 1612.04473$].$

[19] C. Deaconu et al., Measurements and Modeling of Near-Surface Radio Propagation in Glacial Ice and Implications for Neutrino Experiments, Phys. Rev. D 98, 043010 [arXiv: 1805.12576 ].

[20] M. G. Aartsen et al., Multi-messenger observations of a flaring blazar coincident with high-energy neutrino IceCube-170922A, Science 361, 147 [arXiv: 1807 . 08816]. 